OPEN ACCESS

Edited by:

Laura Maria Vergani,

Politecnico di Milano, Italy

Reviewed by:

Walter Caseri,

ETH Zürich, Switzerland Abu Zayed M. Saliqur Rahman,

The Ohio State University, United States

*Correspondence:

Thomas Willett

thomas.willett@uwaterloo.ca

Specialty section:

This article was submitted to Polymeric and Composite Materials,

a section of the journal

Frontiers in Materials

Received: 29 June 2019 Accepted: 10 October 2019 Published: 25 October 2019

Citation: Comeau PA and Willett T (2019) An Alternative Approach to the Surface Methacrylation of Non-stoichiometric Hydroxyapatite Nanoparticles for Use in Bone-Inspired Composites. Front. Mater. 6:263

doi: 10.3389/fmats.2019.00263

\section{An Alternative Approach to the Surface Methacrylation of Non-stoichiometric Hydroxyapatite Nanoparticles for Use in Bone-Inspired Composites}

\author{
Patricia A. Comeau and Thomas Willett* \\ Composite Biomaterial Systems Laboratory, Department of Systems Design Engineering, University of Waterloo, Waterloo, \\ ON, Canada
}

Modification of the inorganic particle surface is one approach toward enhancing the mechanical and swelling properties of a bone-inspired composite. In this study we sought to manipulate the surface of non-stoichiometric $\mathrm{nHA}$ particles by utilizing a gentler methacrylation approach with two different methacrylates - methacrylic anhydride (MAh) and glycidyl methacrylate (GMA). While silanization of $\mathrm{nHA}(\mathrm{Si}-\mathrm{nHA})$ is a recognized functionalization approach, one notable disadvantage is the need for relatively harsh reaction conditions. Here, ATR-FTIR and liquid state ${ }^{13} \mathrm{C}-\mathrm{NMR}$ provided evidence for a stronger affinity of the $\mathrm{nHA}$ surface for methacrylic anhydride compared to glycidyl methacrylate under the same reaction conditions; fewer GMA molecules reacted with the $\mathrm{nHA}$ surface. However, the affinity of MAh is more electrostatic in nature, whereas GMA attachment involves some covalent bond formation. In addition, while in water, there was no detectable dependence of particle settling on methacrylate choice, in ethanol, native $\mathrm{nHA}$ and $\mathrm{nHA}$ methacrylated with GMA each had detectably greater stability in suspension than the other sample groups. Lastly, the addition of GMA-nHA detectably increased the dynamic stiffness and did not impact the swelling in $37^{\circ} \mathrm{C}$ water of a GelMA-based composite compared to that observed upon adding native nHA. Overall, the GMA-based methacrylation approach was found to be a viable alternative to silanization and offers possibilities for future fabrication of bone-inspired composites.

Keywords: nano-hydroxyapatite, methacrylation, methacrylic anhydride, glycidyl methacrylate, surface functionalization, nanocomposite, dynamic stiffness, swelling

\section{INTRODUCTION}

As an osteoconductive ceramic which resembles the inorganic component of bone, hydroxyapatite (HA; $\left.\mathrm{Ca}_{10}\left(\mathrm{PO}_{4}\right)_{6}(\mathrm{OH})_{2}\right)$ is interesting to biologists and biomaterial scientists alike. To date, HA has found use in applications such as a bone filler (Frame et al., 1981), orthopedic implant coating (Liu et al., 2009), composite filler (Liu et al., 2013), and cell culture substrate (Cheng et al., 2013). In many of these cases, the ability to control or manipulate the surface properties of HA is very important and informs how successful the application will be. For example, the incorporation of HA in bone cement formulations has previously been found to reduce the strength of these 
materials (Leite Ferreira et al., 2014); this is likely due to a poor interfacial bonding between the polymeric matrix and HA, as well as agglomeration of the HA leading to stress concentrations (Leite Ferreira et al., 2014). It is well-recognized that interfacial adhesion between organic matrix and inorganic fillers is one attribute which contributes to the successful development of a composite with good mechanical properties (Ji et al., 2003; Xie et al., 2004). To address this challenge, coupling agents may be used to modify the HA surface and tune conditions at the composite interface (Vaz et al., 2002; Roether and Deb, 2004), including the ability to disperse the HA within the chosen organic matrix. Fortunately, several groups have found that HA may strongly interact with both polar and polarizable molecules, including various proteins and cells (Redey et al., 1999; El-Ghannam, 2005; Pieters et al., 2010; Gamelas and Martins, 2015). As one class of coupling agents, methacrylates covalently attached to a particle surface provide an opportunity for selective radical reactions within a composite. Methacrylated groups can aid in the covalent cross-linking between inorganic and organic phases within a composite; this can be very beneficial in the processing and application of nanocomposites in various industries, including for the biomedical materials and devices industries. Grafting of HA micro-particles with methacrylates has been previously achieved using a redox initiating system with some success (Murugan and Panduranga Rao, 2003; Murugan and Ramakrishna, 2004). However, the gentler approach offered in this study should generate fewer potentially cytotoxic byproducts (or unreacted residual chemicals) (Temenoff et al., 2003). In addition, our proposed approach is expected to be more controlled than a redox initiation system owing to the lack of radicals (or growing macroradicals) which may terminate reactive sites (Murugan and Panduranga Rao, 2003). In this paper, we first present the results of direct functionalization of non-stoichiometric HA nanoparticle (nHA) surfaces using two different methacrylates under mild conditions-methacrylic anhydride (MAh), and glycidyl methacrylate (GMA)-and subsequently report changes to nHA particle dispersion in different media, before adding the nHA to methacrylated gelatin (GelMA) and assessing the composite stiffness and swelling in a proof-of-concept investigation. A surface functionalization control for this study is the well-studied silanated-nHA (Si-nHA), achieved using methacryloxypropyltrimethoxysilane (MPTS) (Cisneros-Pineda et al., 2014; Lung et al., 2016). Unfortunately, typical silanization reactions involve harsh reaction conditions (e.g., acidic $\mathrm{pH}$ ), and a high risk of forming unstable multilayers (Liu et al., 2001; Halvorson et al., 2003; Amdjadi et al., 2017), neither of which is ideal toward the development of an nHAbased bone-mimetic composite.

Crystalline $\mathrm{HA}$ consists of $\mathrm{Ca}^{2+}, \mathrm{PO}_{4}^{3-}$, and $\mathrm{OH}^{-}$groups packed closely together in a hexagonal structure (Valle et al., 2014; Poralan et al., 2015). While $\mathrm{OH}^{-}$serves as the backbone, the 6 phosphate ions helically arranged around the c-axis are responsible for stabilizing the skeletal frame of HA (Poralan et al., 2015). With a $\mathrm{Ca} / \mathrm{P}$ ratio lower than the stoichiometric value for HA (i.e., 1.67), there are calcium vacancies on the surface of non-stoichiometric HA. As a result, the surface calcium atoms are likely covered by excess phosphate ions (El Shafei and
Moussa, 2001; Sarig, 2004; Jahromi et al., 2013) which, when protonated in the aqueous solutions, will produce predominantly $\mathrm{P}-\mathrm{OH}$ groups at the surface. We hypothesized that these surface hydroxyl groups would play a key role in the methacrylation of non-stoichiometric HA nanoparticles (nHA) and aid in the formation of covalent bonds between the nHA surface and the methacrylate agents. Furthermore, the amount of nHA surface methacrylation would also be highly dependent on the type of methacrylate reagent, with glycidyl methacrylate less successful at neutral $\mathrm{pH}$ conditions than methacrylic anhydride. Methacrylic anhydride surface functionalization will be more enhanced owing to a notable side reaction which forms methacrylic acid, reduces the $\mathrm{pH}$, and displaces calcium from the nHA surface; this increases the availability of surface hydroxyl groups available for methacrylation. Meanwhile, the GMA reaction at neutral $\mathrm{pH}$ involves both transesterification (which divides GMA in to methacrylate and glycidyl groups) and epoxide ring opening; together these processes encourage both covalent bonding and electrostatic interactions at the nHA surface.

The formation of agglomerates and particle settling are detrimental to the formation and performance of nanocomposites. Finding a surface functionalization approach which addresses nanoparticle agglomeration and dispersion is key to the development of uniformly mixed and dispersed nanocomposites and their bulk properties for subsequent successful application (Lee et al., 2006). Particles suspended in liquids inherently form aggregates and eventually settle as a result of gravity (Liyanage et al., 2016). A single particle in a static fluid will show a settling rate that is dependent on the density and viscosity of the fluid, as well as the nature of the particle (density, size, shape, surface texture) (Witharana et al., 2012). At higher particle concentrations, some inter-particle interaction must also be considered. In the case of nanoparticles, when dispersed in liquids, the intermolecular forces along with the thermal vibrations and diffusivity play a greater role than Newtonian forces (and gravity). As a result, nanoparticles have a more random (and less vertically downward) motion during settling than particles that are $1 \mu \mathrm{m}$ or larger in size. We hypothesized that the dispersion of the methacrylated particles in different media will be largely dependent on methacrylate reagent type, with each reagent resulting in a different surface charge owing to differences in the nature of surface coverage with the methacrylate agent.

Lastly, a proof-of-concept investigation was pursued to study the impact of nHA surface methacrylation on the dynamic stiffness and swelling of a nHA-added GelMA composite. We hypothesized that successful surface methacrylation of nHA would result in greater composite dynamic stiffness and reduced swelling in water.

\section{MATERIALS AND METHODS}

Non-stoichiometric nHA was purchased from MKNano (division of M K Impex Corp, Canada). All other reagents were purchased from Sigma-Aldrich Canada. We have previously reported on the characteristics of this nHA (Comeau and Willett, 2018). The 
nHA powder has been found to have a crystallinity of $66 \%$, a $\mathrm{Ca} / \mathrm{P}$ of 1.52 and is rod shaped with a $\sim 120 \mathrm{~nm}$ length and $20-$ $30 \mathrm{~nm}$ width on average (Comeau and Willett, 2018). In addition, ATR-FTIR spectroscopy of the raw native nHA has confirmed its carbonated nature (Comeau and Willett, 2018).

\section{Methacrylation of the Non-stoichiometric nHA Surface}

$0.425 \mathrm{M}$ solutions of methacrylic anhydride and glycidyl methacrylate were first prepared using MilliQ distilled water ( $\mathrm{MQ} \mathrm{dH}_{2} \mathrm{O}$ ), and brought to a $\mathrm{pH}$ of 7.4 using $4.0 \mathrm{M}$ sodium hydroxide $(\mathrm{NaOH})$, before $300 \mathrm{~mL}$ of this mixture was added to $12 \mathrm{~g}$ of $\mathrm{nHA}$ within a $1 \mathrm{~L}$ capped flask. The $\mathrm{pH}$ of the nHA-based mixture was then maintained at a $\mathrm{pH}$ of 7.4 using $4.0 \mathrm{M} \mathrm{NaOH}$, while placed on a horizontal shaking plate at $200 \mathrm{rpm}$ for $2 \mathrm{~h}$. After $2 \mathrm{~h}$, the mixture was centrifuged at 2,000 rpm for $10 \mathrm{~min}$, before the liquid was decanted, the powder re-suspended in MQ $\mathrm{dH}_{2} \mathrm{O}$, and the mixture centrifuged for a second time. Once the liquid was decanted for a second time, the nHA powder pellet was manually crushed and placed in a $60^{\circ} \mathrm{C}$ oven for 5 days in order to dry. Figure 1 provides a schematic of the proposed surface reaction between $\mathrm{nHA}$ and either MAh or GMA.

\section{Silanization of the Non-stoichiometric nHA Surface}

First, following a modification of a published protocol (Lung et al., 2016), 30 \% by-volume of methacryloxypropyltrimethoxysilane (MPTS) was added to a $90 \%$ ethanol solution, the $\mathrm{pH}$ was adjusted to 4 with $3.4 \mathrm{M}$ acetic acid, and the mixture was placed under aluminum foil on a magnetic stir plate at $200 \mathrm{rpm}$ for $1 \mathrm{~h}$. Next, the mixture was added to $12 \mathrm{~g}$ of nHA powder in a $1 \mathrm{~L}$ glass capped flask, and the suspension sonicated for $15 \mathrm{~min}$ at $50^{\circ} \mathrm{C}$ in a water bath. The ratio of reactant moles per gram of nHA powder is the same under silanization conditions as under methacrylation conditions (e.g., $0.1275 \mathrm{~mol}$ reactant $/ 12 \mathrm{~g} \mathrm{nHA}$ ). After $15 \mathrm{~min}$, the suspension was returned to the magnetic stir plate at 200 rpm for $24 \mathrm{~h}$. Finally, the Si-nHA suspension was dialyzed for $2 \mathrm{~h}$ against MQ $\mathrm{dH}_{2} \mathrm{O}$, before being collected and dried at room temperature for several days. Owing to the high concentration of MPTS, this powder also required additional rinsing with $100 \%$ ethanol and drying in a furnace for 1 week at $60^{\circ} \mathrm{C}$.

\section{Functionalized nHA Powder Characterization}

The nHA powder was analyzed by Attenuated Total Reflectance - Fourier Transform Infrared (ATR-FTIR) spectroscopy (Tensor 27 , Bruker) to confirm the presence of methacrylate groups ( $n$ $=3$ ). From the ATR-FTIR spectra, vibrations corresponding to $\mathrm{PO}_{4}^{3-}$ were identified at $1,028,605$, and $565 \mathrm{~cm}^{-1}$, while bands indicating $\mathrm{C}=\mathrm{O}$ and $\mathrm{C}=\mathrm{C}$ (from the methacrylates) could be found at 1,680-1,740 and 1,600-1,680 $\mathrm{cm}^{-1}$, respectively (Arcís et al., 2002; Gonzalez-McQuire et al., 2004). The peak area for the $\mathrm{C}=\mathrm{C}$ functional group was calculated using DMFit 2010 Software, following normalization to that of the phosphate vibration at $605 \mathrm{~cm}^{-1}$ and subtraction of the similarly normalized native nHA peaks. The $\mathrm{C}=\mathrm{C}$ functionalization is of greatest interest for our research group with regards to future use of a methacrylated nHA in UV-curable nanocomposites. However, the identification of $\mathrm{C}=\mathrm{O}$ in the ATR-FTIR spectra is very relevant for the recognition of how the different reactant species interact with the nHA surface. Additional analysis of the chemical structure of the methacrylated nHA was obtained using liquidstate nuclear magnetic resonance imaging (NMR; Bruker 500 $\mathrm{MHz}$ Ultrashield ${ }^{\mathrm{TM}}$ ) with a ${ }^{13} \mathrm{C}$ probe. Prior to NMR, the $\mathrm{nHA}$ powder was first dissolved in $400 \mathrm{mM}$ ethylenediaminetetraacetic acid (EDTA) and the solution filtered with cheese cloth (to ensure complete dissolution) before adding deutrium oxide $\left(\mathrm{D}_{2} \mathrm{O}\right)$ and placing the sample in liquid NMR tubes. Samples were run at a frequency of $125.77 \mathrm{MHz}$ using a 90-degree pulse of $15 \mu \mathrm{s}$. Spectra were reported in ppm using the reference of methanol in $\mathrm{D}_{2} \mathrm{O}$. Peaks in the ${ }^{13} \mathrm{C}$ NMR spectra were identified using Topspin (Bruker) software. It is important to recognize an important source of error resulting from the hydrolysis of nHA during liquid NMR sample preparation. However, native (i.e., unmodified) nHA was used as a control in both ATR-FTIR and NMR characterization; this helps to mitigate this concern.

Following drying, the surface charge and relative suspension stability of the methacrylated nHA was assessed by characterizing the zeta potential of $n \mathrm{HA}-\mathrm{dH}_{2} \mathrm{O}$ solutions with a zeta potential analyzer (Wallis ${ }^{\mathrm{TM}}$, Cordouan Technologies) and ZetaQ V1.7.0 software $(n=5)$. Sample suspensions of $0.5 \mathrm{mg} / \mathrm{mL}$ nHA were prepared in unbuffered solutions of $\mathrm{MQ} \mathrm{dH}_{2} \mathrm{O}$ or $100 \%$ ethanol (EtOH). MQ $\mathrm{dH}_{2} \mathrm{O}$ and EtOH were the two media chosen owing to their suitability in future biomimetic composite fabrication. Zeta potential is a common method to measure the electrostatic interaction between suspended particles and the overall colloidal stability of the solution (Salopek et al., 1992; White et al., 2007). Typically, a zeta potential magnitude of $>15 \mathrm{mV}$ indicates that the particles will have some beginning stability from electrostatic considerations; however, a magnitude $>30 \mathrm{mV}$ is associated with more apparent (and "medium") suspension stability (Salopek et al., 1992; White et al., 2007).

\section{nHA Particle Gravity Settling Experiments in Polar Liquid}

To further assess the ease of dispersion and likelihood of particle settling in different liquids, $0.1 \mathrm{~g}$ of each $\mathrm{nHA}$ powder was added to a $20 \mathrm{~mL}$ glass scintillation vial and $20 \mathrm{~mL}$ of liquid (either MQ $\mathrm{dH}_{2} \mathrm{O}$ or $100 \%$ ethanol) was added to match zeta potential measurement conditions. This concentration is chosen as it is close to the upper limit for inter-particle interference in light scattering and imagining techniques (Witharana et al., 2012). Next, an ultrasonicator with a micro-horn (Symphony ${ }^{\mathrm{TM}}$, VWR) was used to initially disperse the powder for $30 \mathrm{~s}$ (30\% power setting). Immediately following the completion of sonication (i.e., time of $0 \mathrm{~s}$ ), a digital image of the powder suspension was recorded using a Canon (Powershot Digital ELPH) camera. The suspension was then left undisturbed overnight with images taken at 1,2, 5, 10, 20, and $30 \mathrm{~min}$, as well as at $24 \mathrm{~h}$.

After $30 \mathrm{~min}$ of gravity settling, $5 \mu \mathrm{L}$ of particle-suspended solution $\left(0.1 \mathrm{~g}\right.$ in $20 \mathrm{~mL} \mathrm{MQ} \mathrm{dH}_{2} \mathrm{O}$ or $\left.\mathrm{EtOH}\right)$ was removed 


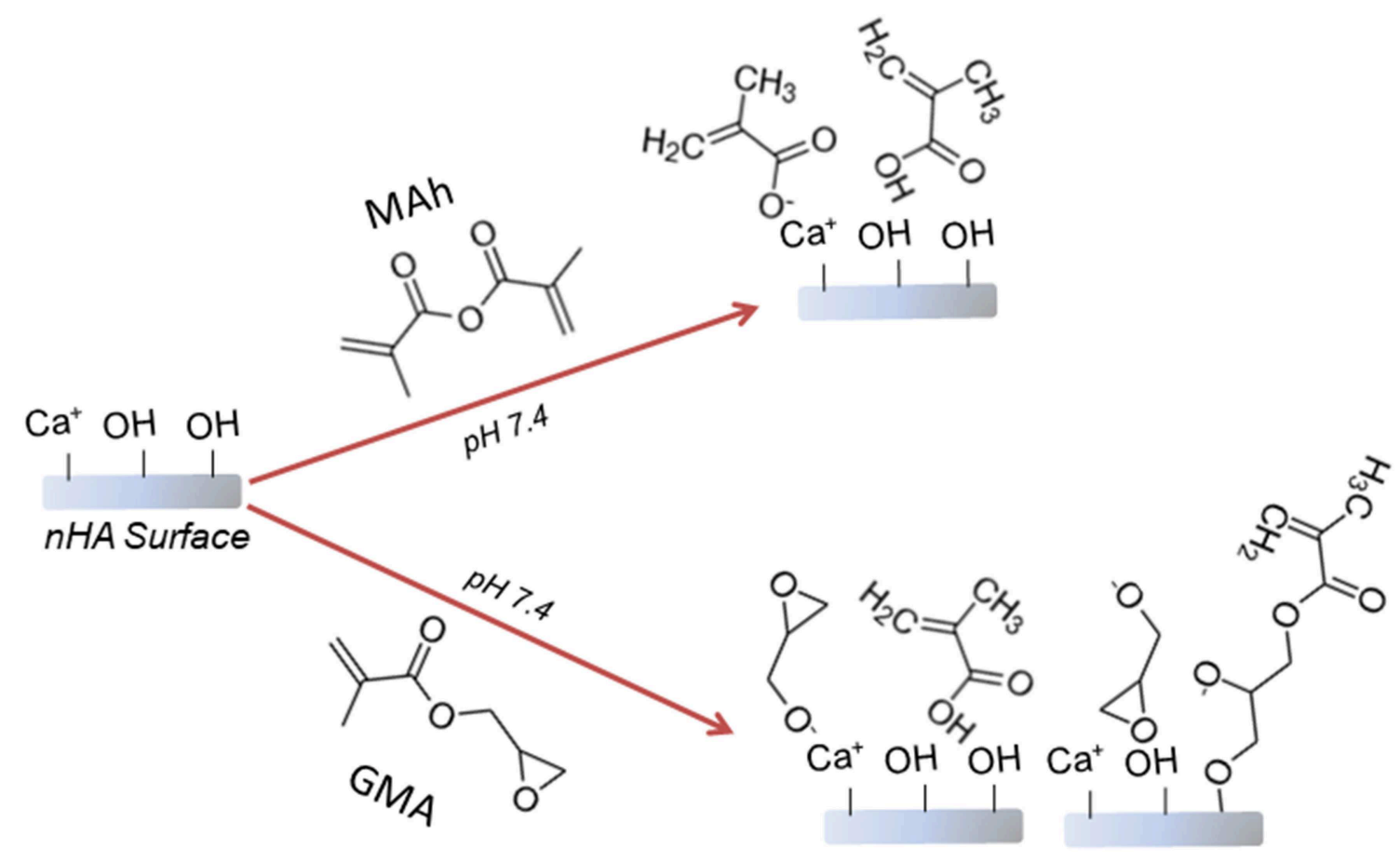

FIGURE 1 | Proposed reaction schematic for surface methacrylation of $\mathrm{nHA}$ with (top) methacrylic anhydride (MAh), and (bottom) glycidyl methacrylate (GMA).

from the vials at the middle of the volume, and added to SEM stubs using carbon tape. The stubs were then left in a desiccator with Drierite ${ }^{\circledR}$ for $24 \mathrm{~h}$ in order to dry prior to SEM imaging (Zeiss Merlin FESEM 1530). Operating conditions for SEM consisted of a working distance of $10.5 \mathrm{~mm}$, an accelerating voltage of $10.00 \mathrm{kV}$, and a vacuum of $1.21 \mathrm{e}^{-006}$ mbar.

\section{Fabrication of nHA-Added GelMA Composite}

Type B, 225 bloom gelatin was methacrylated according to an existing protocol. Gelatin ( $25 \mathrm{~g}$ ) was slowly added to $40^{\circ} \mathrm{C}$ MilliQ distilled water $\left(\mathrm{MQ} \mathrm{dH}_{2} \mathrm{O} ; 100 \mathrm{~mL}\right)$ and the $\mathrm{pH}$ then adjusted to 7.40 using sodium hydroxide $(\mathrm{NaOH} ; 4 \mathrm{M})$ solution. Next, methacrylic anhydride was added while maintaining the $\mathrm{pH}$ at 7-7.4 with $\mathrm{NaOH}(4 \mathrm{M})$. Sufficient methacrylic anhydride was added to obtain a methacrylic anhydride-to-amine group ratio of 10:1 during reaction. Over the next $2 \mathrm{~h}$ the reaction was continued while maintaining the $\mathrm{pH}$ at $7-7.4$ with $\mathrm{NaOH}$ (4 M). After $2 \mathrm{~h}$, the $\mathrm{pH}$ of the gelatin solution was adjusted to 8 with $\mathrm{NaOH}(4 \mathrm{M})$, before dialyzing the methacrylated gelatin (GelMA) solution with $13 \mathrm{kDA}$ cut-off tubing against MQ $\mathrm{dH}_{2} \mathrm{O}$ for seven days. The GelMA solution was then frozen overnight and subsequently freeze dried (Labconco Freezone 1L Benchtop) for 2-3 days to obtain dried GelMA. Finally, GelMA-based inks containing 8\%/vol nHA were prepared by adding nHA (MKNano, MK Impex Corp.) to an aqueous $62 \%$ w/v GelMA solution using a mechanical mixer $\left(\right.$ Caframo ${ }^{\circledR}$ ) at $60 \mathrm{rpm}$.

\section{Dynamic Mechanical Analysis of nHA-Added GelMA Composite}

To obtain viscoelastic properties of the $30 \mathrm{~s}$ UV-cured nHAGelMA samples (of $\sim 4.8 \mathrm{~mm}$ diameter and $\sim 4.5 \mathrm{~mm}$ height), the fresh samples were preloaded in compression to $0.1 \mathrm{~N}$ before subjecting them to cyclic sinusoidal loading between 0 and $10 \%$ compressive strain at a frequency of $0.1 \mathrm{~Hz}$ using a $50 \mathrm{~N}$ loading cell (UniVert, CellScale, Waterloo, Ontario, Canada). Data was acquired at $100 \mathrm{~Hz}$ and analyzed using Matlab code. From this analysis, the dynamic modulus $\left(E^{*}\right)$, storage modulus $\left(E^{\prime}\right)$, and loss modulus $\left(E^{\prime \prime}\right)$ were determined $(n=6)$.

\section{Swelling of nHA-Added GelMA Composite in Water}

To assess the swelling of freshly prepared nHA-GelMA composite samples, the mass of each sample (again, cured for $30 \mathrm{~s}$ ) was first recorded (" $m_{1}$ ") and then added directly to $37^{\circ} \mathrm{C} \mathrm{MQ} \mathrm{dH}_{2} \mathrm{O}$. After 3 days, the mass of the samples was recorded (" $\mathrm{m}_{2}$ "). The swelling percentage of the UV-cured samples in water was calculated according to Equation $(1)(n=6)$.

$$
\frac{\left(m_{2}-m_{1}\right)}{m_{1}} \times 100 \%
$$

\section{Statistical Analysis}

Differences in the means of study outcomes were analyzed using IBM $^{\circledR}$ SPSS $^{\circledR}$ Statistics software and a one-way or two-way general linear model with a significance value of $p=0.05$ (and a post-hoc Tukey analysis). Specific $p$-values for given data sets are 


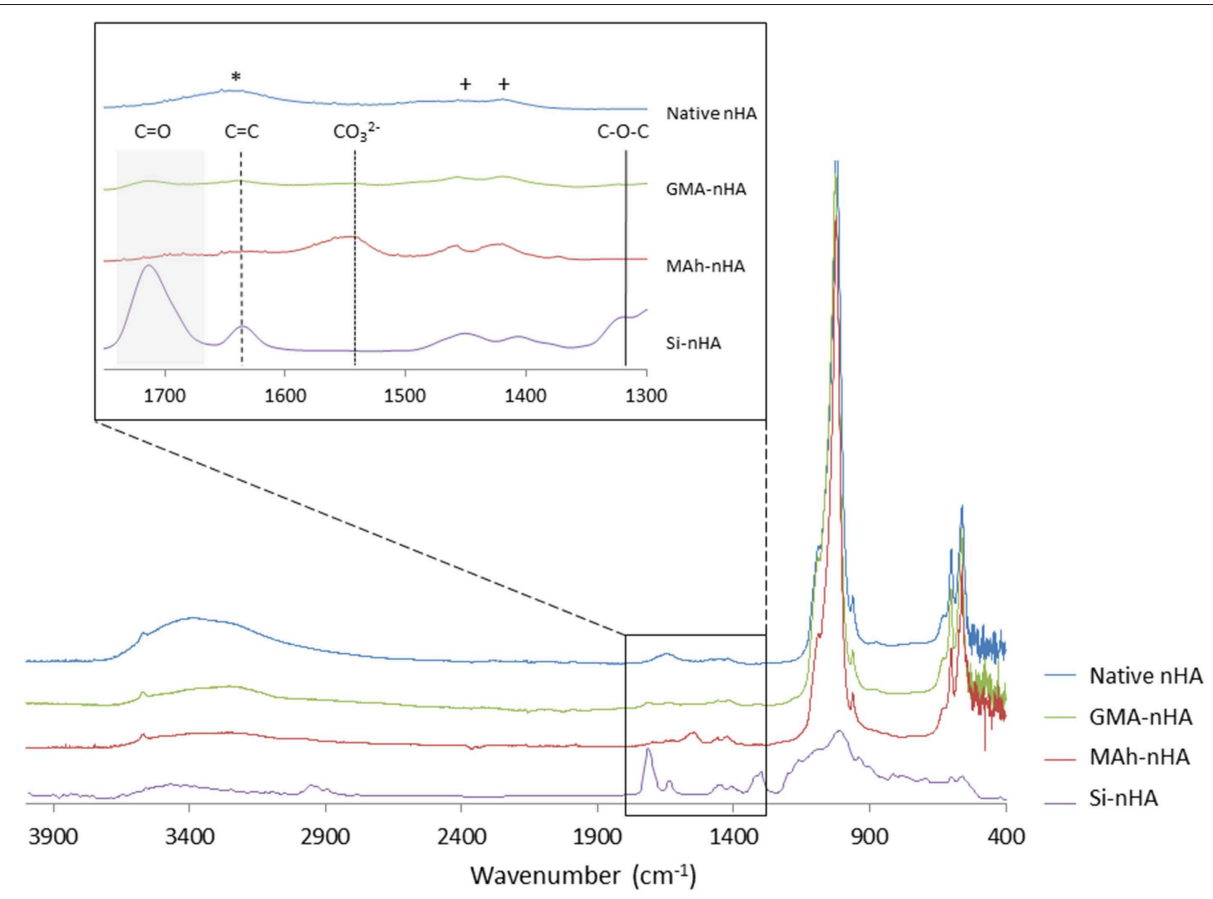

FIGURE 2 | ATR-FTIR spectroscopy of native and methacrylated nHA particles. In this study, nHA was methacrylated using methacrylic anhydride (MAh) or glycidyl methacrylate (GMA). Phosphate peaks in the fingerprint region of the spectra $\left(500-1,200 \mathrm{~cm}^{-1}\right)$ are evident, with spectra of methacrylated $\mathrm{nHA}$ also presenting additional peaks. Peaks owing to $\mathrm{C}=\mathrm{C}$ stretch are identified by dashed vertical lines and $\mathrm{C}=\mathrm{O}$ broad stretch peak by a gray region for the functionalized $\mathrm{nHA}$ samples. The peaks identifying carbonate groups that have replaced tetrahedral phosphate sites are indicated by ${ }^{+}$, while a free residual water $(\mathrm{H}-\mathrm{O}-\mathrm{H})$ peak is indicated by * in the native $\mathrm{nHA}$ spectra.

provided (noting that $p$-values $<0.05$ are significant). All data are presented in this paper as mean \pm one standard deviation.

\section{RESULTS AND DISCUSSION Characterization of Functionalized nHA Powders}

nHA particles were functionalized using the two methacrylatesmethacrylic anhydride (MAh) and glycidyl methacrylate (GMA). A $0.425 \mathrm{M}$ concentration of methacrylate reagents was chosen to encourage full saturation of the nHA surface with excess remaining in solution following reaction. This methacrylate reagent concentration also closely matches the previously reported study of GMA-functionalization of HA micrometersized particles using a redox-initiating system (Murugan and Panduranga Rao, 2003; Murugan and Ramakrishna, 2004). Meanwhile, these two methacrylates were chosen for their different chemical structure and potential differences in bonding to the nHA surface upon reaction (e.g., electrostatic or covalent in nature). ATR-FTIR spectroscopy (Figure 2) of the two methacrylated powders shows evidence of additional peaks (compared to native nHA) that correspond to the presence of methacrylate groups.

ATR-FTIR spectroscopy confirmed the carbonatesubstitution of the native, non-stoichiometric nHA with peaks at 1,423 and $1,457 \mathrm{~cm}^{-1}$ attributed to asymmetric stretching modes of carbonate groups which replaced the tetrahedral phosphate sites (Figueiredo et al., 2012; Tkalčec et al., 2014). The broad peak at $1,642 \mathrm{~cm}^{-1}$ for native $\mathrm{nHA}$ is attributed to free residual water (H-O-H) (Zou et al., 2012), which following methacrylation is less evident in the MAh-nHA and GMA-nHA samples. Several additional peaks also appear in the spectra following methacrylation of the nHA. For example, the band at $1,566 \mathrm{~cm}^{-1}$ for MAh-nHA is representative of the carboxylate group formed during the ionic side reaction between methacrylic acid and calcium ions at the nHA surface (Cisneros-Pineda et al., 2014); this suggests a large amount of electrostatic interactions at the nHA surface. In addition, $\mathrm{C}=\mathrm{O}$ and $\mathrm{C}=\mathrm{C}$ bond stretches are apparent for each methacrylate reagent; these are indicative of at least the presence of these reagents at the nHA surface (Arcís et al., 2002; Cisneros-Pineda et al., 2014). For MAh-nHA, the $\mathrm{C}=\mathrm{O}$ peak occurs at $1,696 \mathrm{~cm}^{-1}$, while the $\mathrm{C}=\mathrm{C}$ peak occurs at $1,634 \mathrm{~cm}^{-1}$. For GMA-nHA, these same peaks occur at 1,715 and $1,640 \mathrm{~cm}^{-1}$, respectively. In addition, for MAh-nHA and GMA-nHA, the $\mathrm{C}=\mathrm{C}$ and $\mathrm{C}=\mathrm{O}$ peak areas are similar (data for $\mathrm{C}=\mathrm{O}$ peak fitting not shown) - this is likely indicative that the $\mathrm{COO}^{-}$functional group is not the sole form of attachment for the respective reagents at the nHA surface. Finally, GMA-nHA spectra show a band at $\sim 1,300 \mathrm{~cm}^{-1}$ which is attributable to the $\mathrm{C}-\mathrm{O}-\mathrm{C}$ bond present in the respective reagent; notably MAh-nHA spectra does not show such a peak (Cisneros-Pineda et al., 2014). Meanwhile, the reduced peak area under $C=C$ peaks for GMA-nHA compared to MAh-nHA suggests that 


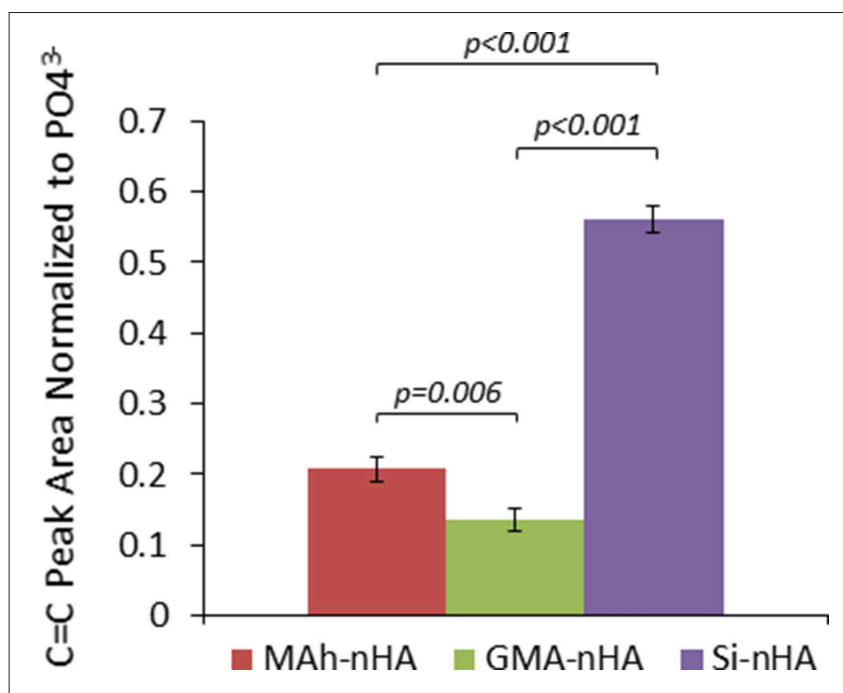

FIGURE 3 | ATR-FTIR analysis of the methacrylated $\mathrm{nHA} C=\mathrm{C}$ peak area normalized to $\mathrm{PO}_{4}^{3-}$ peak area. Horizontal bars indicate statistically detectable difference ( $p$-values indicated) $(n=3)$.

fewer methacrylate groups are present at the nHA surface for the GMA-nHA group (Figure 3).

The difference in means between sample groups was statistically detectable for normalized $\mathrm{C}=\mathrm{C}$ peak area $(p<$ 0.001). For example, GMA-nHA was found to have detectably less $\mathrm{C}=\mathrm{C}$ signal than MAh-nHA $(p=0.006)$. Meanwhile, upon normalization of the $C=C$ peak area to that of the $605 \mathrm{~cm}^{-1}$ phosphate peak, it is evident that the silanization protocol (Lung et al., 2016) resulted in detectably more $\mathrm{C}=\mathrm{C}$ bonds at the surface of Si-nHA than nHA functionalized with either methacrylate reagent $(p<0.001)$. Such $\mathrm{C}=\mathrm{C}$ bonds are integral to the radical photopolymerization reaction; introducing these groups on the nHA surface should encourage radical photopolymerization with similar groups within the GelMA matrix (once nHA and GelMA are blended together in a bone-inspired composite).

Liquid-state ${ }^{13} \mathrm{C}$-NMR spectra further support this difference between the different functionalization approaches and gives insight in to the nature of bonding and/or surface interaction with the different reagents (Figure 4).

The ${ }^{13} \mathrm{C}$-NMR spectra confirm silanization or methacrylation of the nHA following the respective protocols. The highlighted peaks [at (i)-(iv)] are detected in the spectra of MAh-nHA and GMA-nHA, but not in that of native nHA. For example, at 120-145 ppm (peak "ii"), there are two strong peaks for MAh-nHA and GMA-nHA that indicate the presence of vinyl carbon atoms (i.e., $=\mathrm{CR}_{2}$ and $=\mathrm{CH}_{2}$ ) (Reis et al., 2009; Mertz et al., 2014). For MAh-nHA the $=\mathrm{CR}_{2}$ peak has been shifted downfield (i.e., more positive ppm) to $142 \mathrm{ppm}$, while for GMA-nHA this same peak is at $127 \mathrm{ppm}$. This difference in peak position is likely due to greater van der Waals attractive interactions between MAh and nHA (Li and Chesnut, 1986; Chesnut et al., 1988). The $=\mathrm{CH}_{2}$ peak is at the same position (i.e., $120 \mathrm{ppm}$ ) for MAh-nHA and GMA-nHA. Meanwhile, peaks between 160 and 180 ppm (peak "i") in all spectra are associated with carbonyl carbon atoms (Mertz et al., 2014), and the presence of a peak at 70 ppm (peak "iii") in GMA-nHA supports the presence of a C-O-P covalent bond (Avci and Mathias, 2005). Interestingly, there is no apparent $\mathrm{C}-\mathrm{O}-\mathrm{P}$ peak in ${ }^{13} \mathrm{C} \mathrm{NMR}$ spectra for MAh-nHA; this suggests that while functionalization results in a covalent bond between GMA and nHA, this is not the case for MAh. Therefore, MAh interaction with the surface of nHA may be more electrostatic in nature. Lastly, methylene carbon atoms are likely represented by the peak at 20 ppm (peak "iv"). Based on a larger vinyl carbon signal, as well as the additional peaks in ${ }^{13} \mathrm{C}$-NMR spectra of MAhnHA compared to that of GMA-nHA, the methacrylation with methacrylic anhydride results in more methacrylate groups being present at the nHA surface following functionalization; however, these are not covalently bonded to nHA. This is corroborated with a stronger $\mathrm{C}=\mathrm{C}$ peak in ATR-FTIR spectra for MAhnHA compared to GMA-nHA (Figures 1, 2). Altogether, the ATR-FTIR and ${ }^{13} \mathrm{C}$ NMR results support the initial hypothesis that methacrylic anhydride surface functionalization will be more successful with a greater abundance of vinyl groups, owing to the formation of methacrylic acid and the increased presence of hydroxyl groups at the nHA surface. Meanwhile, GMA functionalization involves notable covalent bonding (as a result of epoxide ring opening) and non-covalent interactions (resulting from hydrogen bonding and interactions with $\mathrm{Ca}^{2+}$ at the nHA surface). A notable limitation of liquid ${ }^{13} \mathrm{C}-\mathrm{NMR}$ analysis is the use of ethylenediaminetetraacetic acid (EDTA) to dissolve the nHA, as this creates additional carbon peaks in the spectra. However, identification of peaks due to EDTA and native non-stoichiometric nHA aided in distinguishing those peaks due to the surface functionalization process alone. This was found to be an acceptable approach for processing the liquid NMR spectra and assessing surface functionalization success; particularly when compared with ATR-FTIR spectroscopy results.

Prior to performing settling experiments, the powder was assessed with a Zeta Potential Analyzer in unbuffered MQ $\mathrm{dH}_{2} \mathrm{O}$ and $\mathrm{EtOH}$. There was a noticeable dependence of the zeta potential of the particles on the choice of methacrylating agent in either of the two unbuffered media (Figure 5).

The impact of sample type and solution type on zeta potential was statistically detectable ( $p=0.015$ and $p<0.001$, respectively). In fact, the interaction between sample type and solution type was also found to be significant ( $p<0.001$ ). Both MAh-nHA and $\mathrm{Si}-\mathrm{nHA}$ were found to have a detectably less negative zeta potential in $\mathrm{MQ} \mathrm{dH}_{2} \mathrm{O}$ than GMA-nHA $(p=0.035$ and $p<0.001$, respectively). Meanwhile, in $\mathrm{EtOH}, \mathrm{MAh}-\mathrm{nHA}$ and Si-nHA were found to have a detectably less positive zeta potential than either native nHA or GMA-nHA ( $p<0.001$ for each respective pair).

To explain the zeta potential dependence on methacrylating reagent, the reactions which are uniquely specific to each reagent at the nHA surface must be considered. For example, upon methacrylating nHA with methacrylic anhydride, a side reaction between methacrylic anhydride and water produces methacrylic acid. These methacrylic acid molecules are able to stick to the nHA surface and, subsequently, release additional protons into the solution. Meanwhile, methacrylating nHA with glycidyl methacrylate itself involves additional interactions between the glycidyl groups (formed during the transesterification of glycidyl 


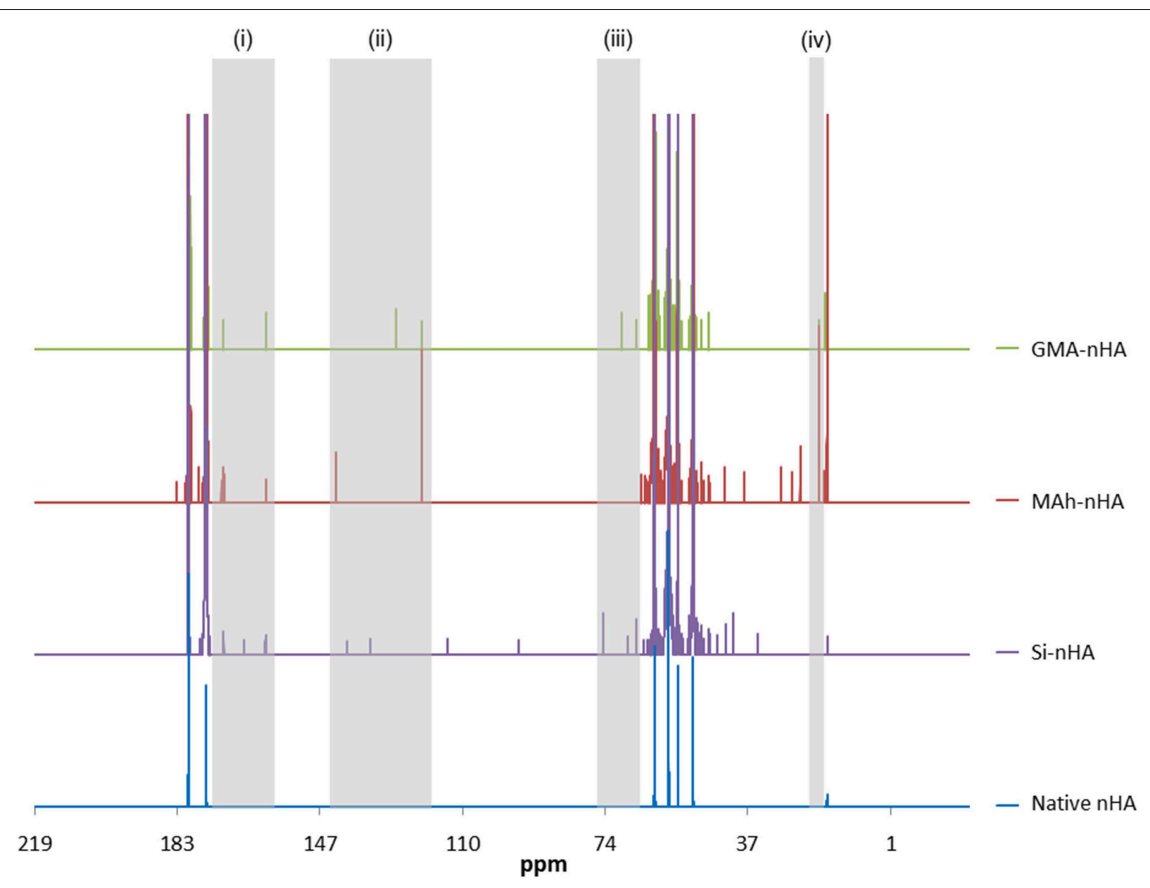

FIGURE 4 | Liquid state ${ }^{13} \mathrm{C}$ NMR spectra of native, silanated and methacrylated nHA. In this study, nHA is methacrylated using methacrylic anhydride (MAh) or glycidyl methacrylate (GMA). There are several peaks labeled (i)-(iv) in the Si-nHA, MAh-nHA and GMA-nHA spectra which do not appear in the native nHA spectrum.

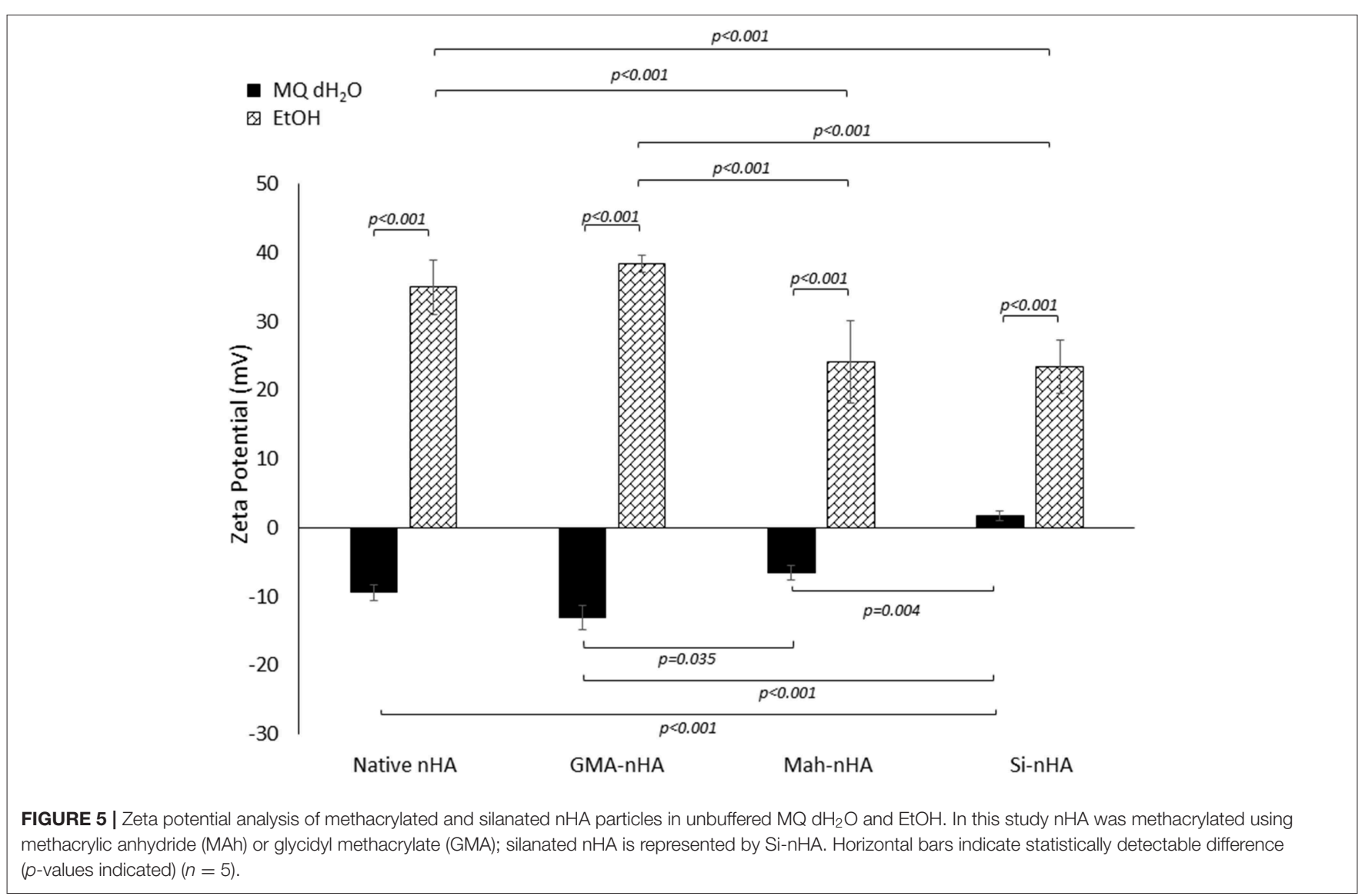




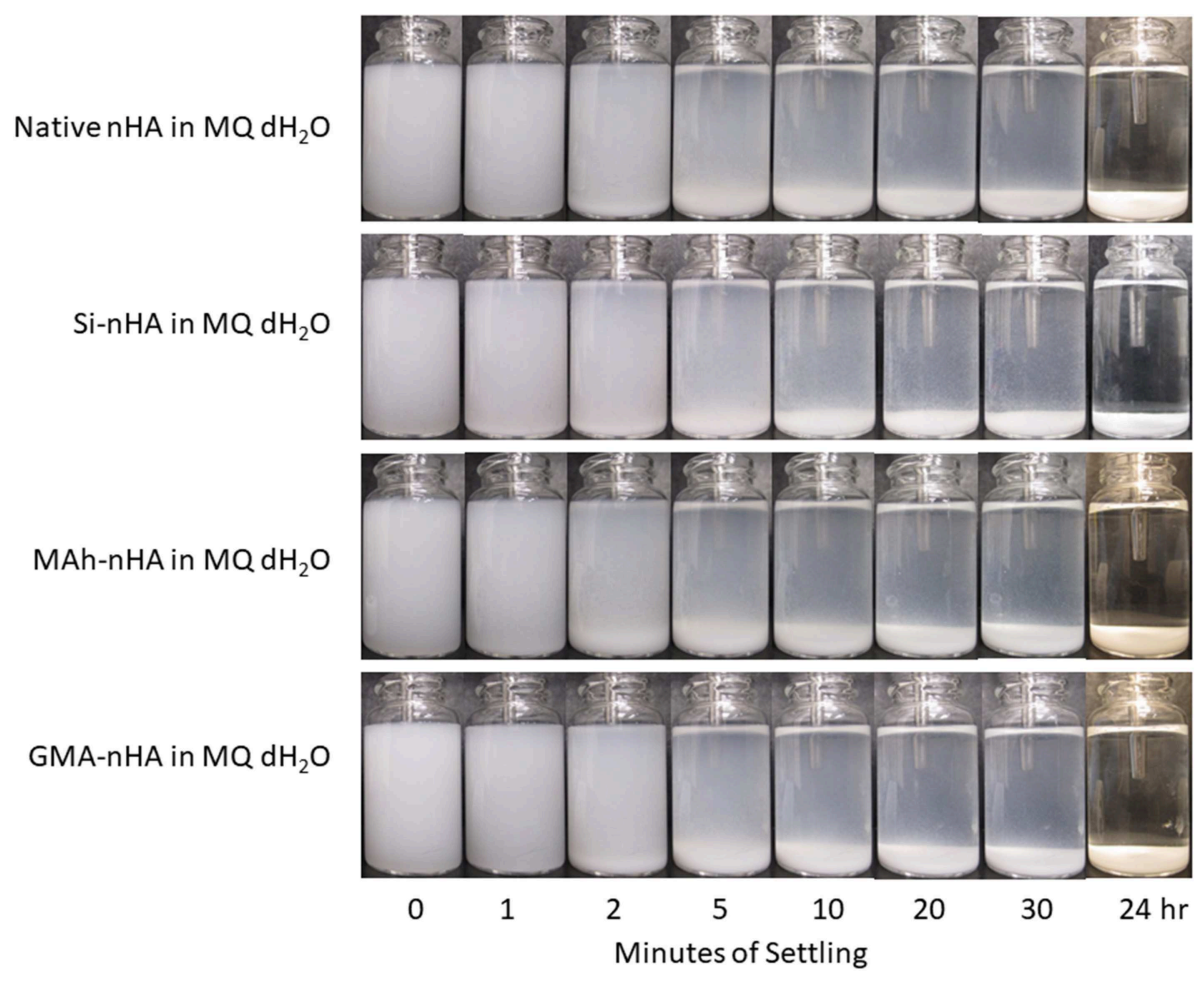

FIGURE 6 | Gravity settling of native, methacrylated, and silanated $\mathrm{nHA}$ in $\mathrm{MQ} \mathrm{dH}_{2} \mathrm{O}$ at room temperature. In this study, $\mathrm{nHA}$ is methacrylated using methacrylic anhydride (MAh) or glycidyl methacrylate (GMA); silanated $\mathrm{nHA}$ is represented by Si-nHA.

methacrylate) and the hydroxyl groups and/or positive charge (e.g., $\mathrm{Ca}^{2+}$ ) at the nHA surface. Altogether, the zeta potential of the two methacrylated nHA supports a theory of less glycidyl methacrylate bonding to the hydroxyl groups at the nHA surface (and less coverage of the negative surface charge reported for unmodified nHA). In fact, there is no statistically detectable difference in zeta potential between native nHA and GMA-nHA in either media measured. From the zeta potential measurements alone, it would then be anticipated that none of the sample groups will exhibit appreciable particle stability (i.e., suspension) in MQ $\mathrm{dH}_{2} \mathrm{O}$. Meanwhile, in EtOH native nHA and GMAnHA particles show more particle stability than either Si-nHA or MAh-nHA particles.

There are a number of mechanisms responsible for developing charge - the three of notable relevance here are (1) ionization of surface groups, (2) differential ion adsorption from electrolyte solution, and (3) differential ion dissolution from a crystal lattice. For example, water is dissociated to a larger extent than alcohols (e.g., ethanol) (Logtenberg and Stein, 1986). As a result, a greater concentration of $\mathrm{H}^{+}$and $\mathrm{OH}^{-}$are available to be adsorbed by the nHA surface when in water; this coincides with some dissolution of the positive divalent calcium ion from the nHA lattice and an altogether more negative zeta potential in water. In ethanol $(\mathrm{EtOH})$, there are fewer ions available to adsorb on to nHA, and the calcium ions are less mobile in leaving nHA; this results in a more positive zeta potential. Owing to the silane groups on the Si-nHA, the surface is more hydrophobic (Roether and Deb, 2004), with much lower adsorption of $\mathrm{H}^{+}$and $\mathrm{OH}^{-}$, and the zeta potential is the most positive of the four nHA sample groups upon dispersion in water. This also explains why the difference of Si-nHA zeta potential in water compared to that in ethanol is the smallest (of the four groups studied).

\section{nHA Particle Settling Experiments}

Results from gravity settling experiments of the sample groups in MQ $\mathrm{dH}_{2} \mathrm{O}$ and $\mathrm{EtOH}$ are shown in Figures 6, 7, respectively.

Consistent with the zeta-potential results, the unstable suspension of the sample groups in MQ $\mathrm{dH}_{2} \mathrm{O}$ demonstrates quick settling, particularly during the first $2-5 \mathrm{~min}$ of the experiment. The liquid above the particle bed becomes more transparent after $2 \mathrm{~min}$ for the native nHA and nHA methacrylated with either agent; this suggests more of the particles have settled out. For each sample group, the liquid phase is not completely clear within the first $30 \mathrm{~min}$ in $\mathrm{dH}_{2} \mathrm{O}-$ this is indicative that some small particles remain suspended at each time point. However, $24 \mathrm{~h}$ later, most of the particles have settled to the bottom of the vial. Zeta potential results in $\mathrm{MQ} \mathrm{dH}_{2} \mathrm{O}$ correlate well with the observed particle settling in $\mathrm{dH}_{2} \mathrm{O}$; the magnitude of zeta potential is $<15 \mathrm{mV}$ for each sample (which itself suggests poor nanoparticle suspension in the given media). Meanwhile, the suspensions of all but the Si-nHA sample group particles in ethanol may be considered stable for at least the first $30 \mathrm{~min}$ recorded, as few particles appear to settle. Following $24 \mathrm{~h}$ of settling in ethanol, the MAh-nHA particles have largely settled to the bottom of the vial, while Si-nHA have a few particles still suspended, and 


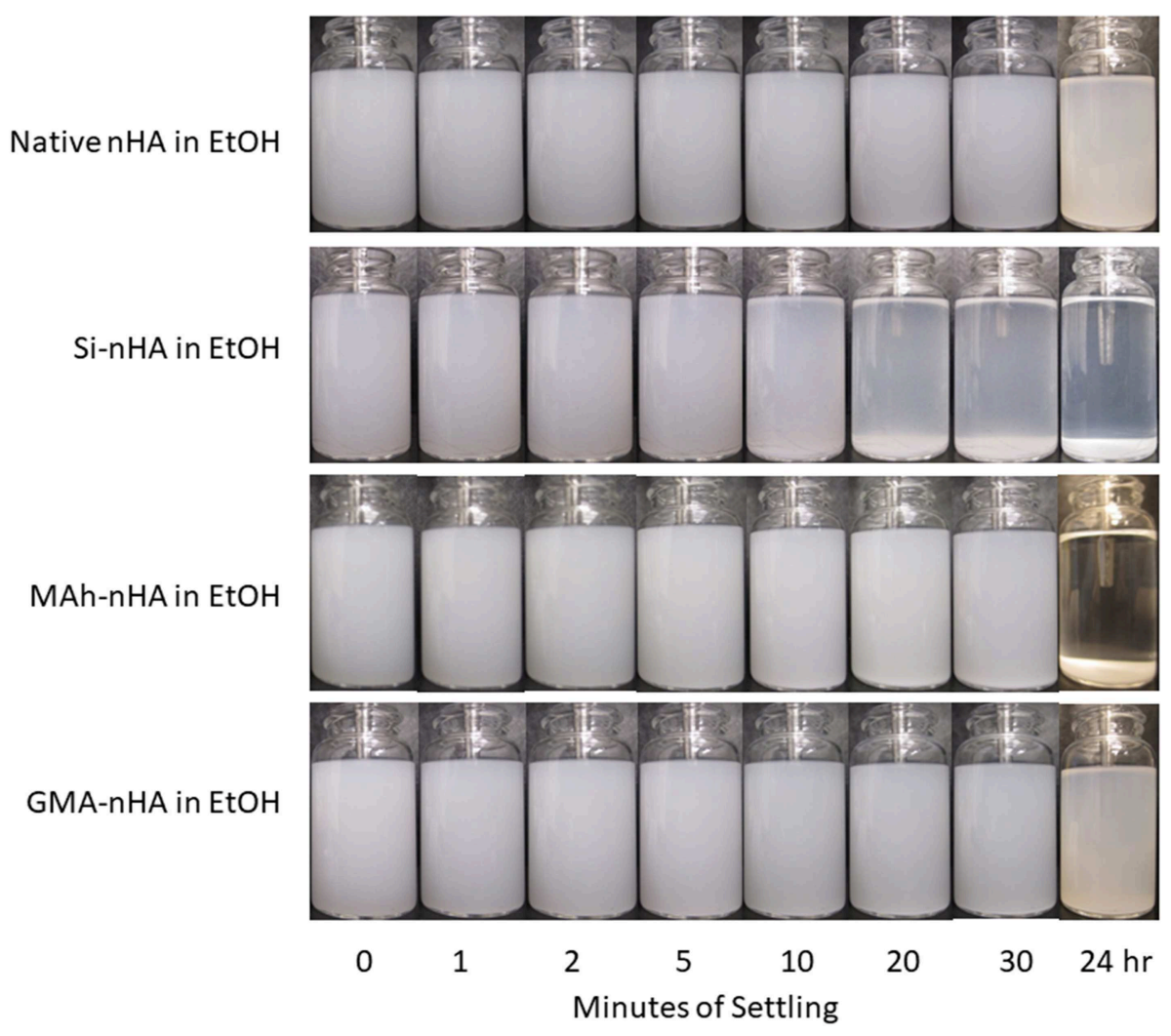

FIGURE 7 | Gravity settling of native, methacrylated, and silanated nHA in 100\% ethanol (EtOH) at room temperature. In this study nHA is methacrylated using methacrylic anhydride (MAh) or glycidyl methacrylate (GMA); silanated $\mathrm{nHA}$ is represented by Si-nHA.

the other sample groups have a significant amount of powder suspended. This is again correlated with the zeta potential results as only native nHA and GMA nHA were found to have a charge $>30 \mathrm{mV}$ in magnitude (and some expected suspension stability) (Salopek et al., 1992; White et al., 2007). The lower the magnitude of zeta potential, the greater the ease of particle approach with other particles (i.e., less inter-particle repulsion). In considering the concentration of nHA in the suspension media, there is considerable room for the particles to move around in either media. The difference based on suspension media then is largely owing to how the particles interact with the media, the nature of the solvent layer, and how the various forces (e.g., attractive, repulsive) balance. A common law for understanding particle settling is Stoke's law. In accordance with this law, as ethanol has a lower density and greater dynamic viscosity than water, it is to be expected that particle settling will occur at a slightly slower rate. However, as Stoke's law minimizes the interactions between particles, these are not the only factors to consider. For example, ethanol $\left(\mathrm{C}_{2} \mathrm{H}_{5} \mathrm{OH}\right)$ has only one polar end which, upon interaction with the surface of nHA, manages to neutralize some of the attractive forces, minimize agglomeration, and improve the overall dispersion of nHA (compared to that observed in water). Of significance is that while native nHA and GMA-modified nHA have similar settling behavior in either media, the GMA-modified nHA also offer
$\mathrm{C}=\mathrm{C}$ groups which should benefit the mechanical and swelling performance of future nHA-added bone-inspired composites. Future study may consider additional quantification of the particle settling behavior qualified here (including additional settling time points), as well as that within the future composite of interest.

Under dry conditions, and using SEM to image the powder following $30 \mathrm{~min}$ of dispersion, the differences observed in zeta potential measurement were less strongly evident owing to a relatively small magnitude of surface charge in most conditions (Figure 8). Typically powder dispersion is qualified by a zeta potential magnitude $>15 \mathrm{mV}$, and while native nHA and GMA-nHA do report such a high magnitude in EtOH, this is not strongly evident in the SEM images. Each sample groups shows some agglomeration, with both methacrylation and silanization protocols appearing to have slightly reduced this agglomeration tendency (compared to native nHA). In addition, while agglomeration is evident in all SEM images the proportion of small, fine particles appears to be greater in samples collected after $30 \mathrm{~min}$ in ethanol than in samples after $30 \mathrm{~min}$ in water. The minimal impact of sample group on short-term dispersion (i.e., up to $30 \mathrm{~min}$ ) is likely indicative of the strong role dispersion media has on nanoparticle settling and the random motion of the nanoparticles themselves (i.e., gravity is not the biggest contributor to settling here). 


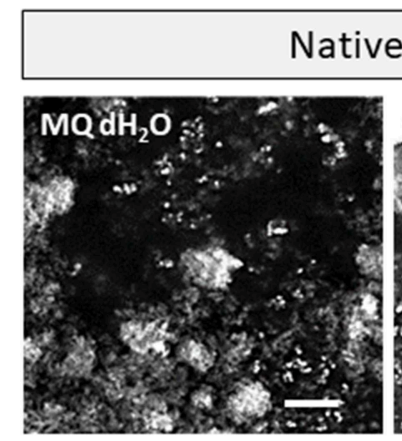

\section{e $\mathrm{HA}$}
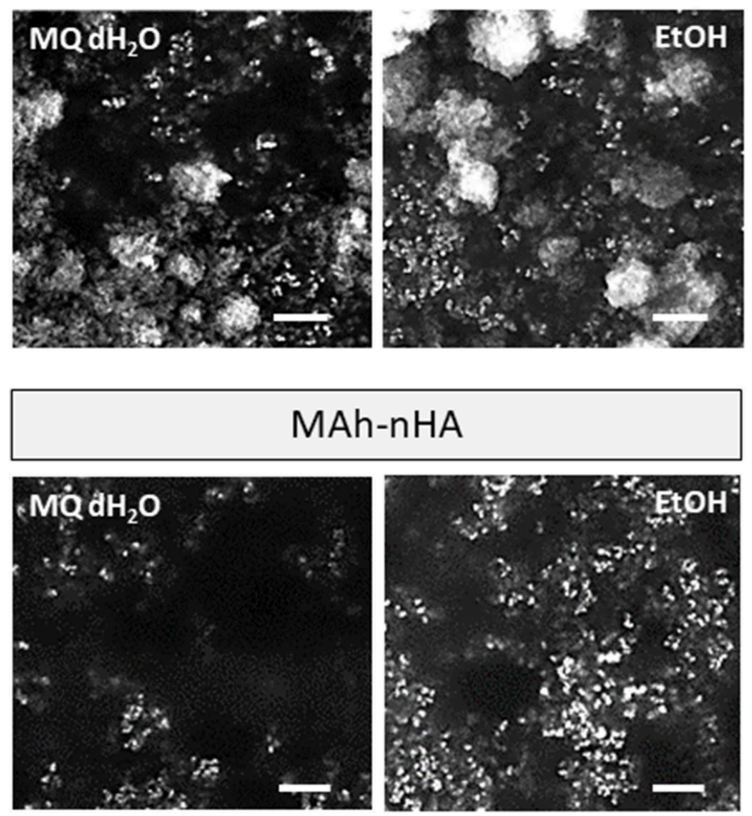

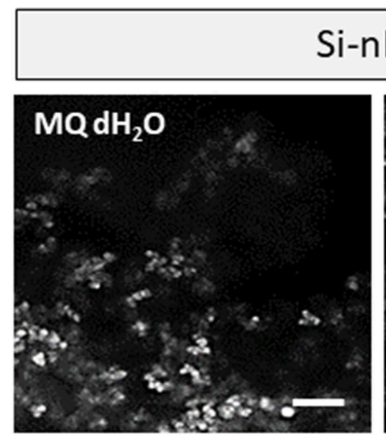

Si-nHA
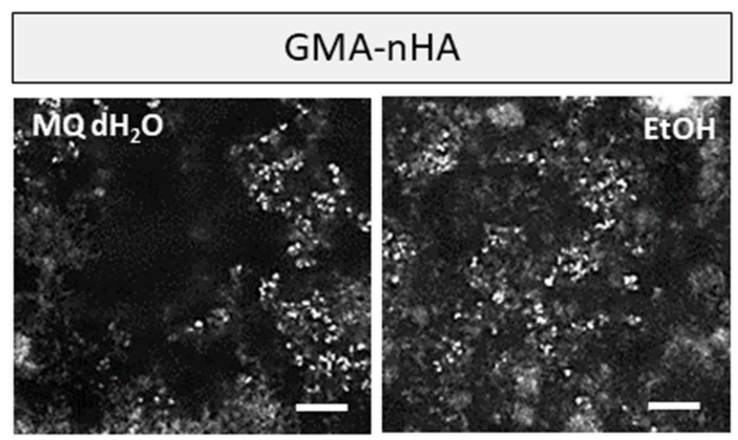

FIGURE 8 | SEM images of native, methacrylated, and silanated $\mathrm{nHA}$ particles with left-side showing particles collected from $\mathrm{dH}_{2} \mathrm{O}$ suspension, and right-side images showing particles collected from EtOH suspension. In this study $\mathrm{nHA}$ is methacrylated using methacrylic anhydride (MAh) or glycidyl methacrylate (GMA); silanated $\mathrm{nHA}$ is represented by Si-nHA. White scale bar represents $1 \mu \mathrm{m}$.

\section{nHA-Added GelMA Composite}

Following characterization of the modified nHA particles alone, the next objective of this study was to investigate the impact of nHA surface methacrylation on the dynamic stiffness and swelling of nHA-added GelMA composite; both of which are integral to the potential application of this material. In this proof-of-concept investigation $8 \% / \mathrm{vol} \mathrm{nHA}$ was dispersed into an aqueous GelMA solution (62\% w/v). Following initial ink processing, it was observed that both MAh-nHA and Si-nHA particles settled out of the GelMA phase relatively quickly, while native nHA remained well-dispersed for at least $30 \mathrm{~min}$ and GMA-nHA experienced only a small amount of settling in this same time period. While all inks were re-mixed immediately prior to preparing the UV-cured samples, this settling behavior is of great significance and correlates well with prior zeta potential observations in an aqueous environment. Meanwhile, a univariate general linear model revealed that the type of surface functionalizing agent impacted dynamic stiffness $\left(\mathrm{E}^{*}\right)$, storage modulus $\left(\mathrm{E}_{\text {storage }}\right)$, loss modulus $\left(\mathrm{E}_{\text {loss }}\right)$, and change in mass during swelling $(p=0.005,0.004,0.014$, and $p<0.001$, respectively; Figure 9).

In fact, of the three nHA-modified GelMA composites, only the GMA nHA-GelMA composite had a detectably greater dynamic stiffness than the native nHA-GelMA composite $(p=$ 0.043). In addition, GMA nHA-GelMA composite swelling after $3 \mathrm{~d}$ in water was comparable to that of native nHA-GelMA composite. Furthermore, the MAh nHA-GelMA composite had the only detectably increased change in mass during swelling compared to either native nHA-GelMA or Si nHA-GelMA composites ( $p=0.001$ and $p<0.001$, respectively). Future studies will need to investigate the dispersion of the surface methacrylated nHA within the GelMA matrix and assess how such dispersion may have impacted the composite properties. In addition, the role that the introduced vinyl groups on the nHA surface have on composite properties, as a result of UV curing (and radical photopolymerization), requires further investigation.

Overall, the processing of nanocomposites suitable for various industries, such as biomedical materials, may be improved with the use of ethanol to improve dispersion of nonstoichiometric nHA, and the inclusion of directly methacrylated non-stoichiometric nHA particles within the composite. In turn, the resulting composite will be better suited to meet requisite properties (e.g., mechanical and swelling) for the given tissue engineering application.

\section{CONCLUSIONS}

In this study, we investigated a new approach to the methacrylation of non-stoichiometric hydroxyapatite nanoparticles (nHA) using two different methacrylates. Methacrylation using methacrylic anhydride (MAh) was found to add more vinyl (i.e., $\mathrm{C}=\mathrm{C}$ ) groups than glycidyl methacrylate (GMA) upon characterization with ATR-FTIR and liquidstate ${ }^{13} \mathrm{C}$-NMR. However, the MAh interaction at the nHA surface was more electrostatic in nature, while for GMA-nHA there was additional evidence of epoxide ring opening and covalent bonding (in the form of P-O-C linkages). Meanwhile, 


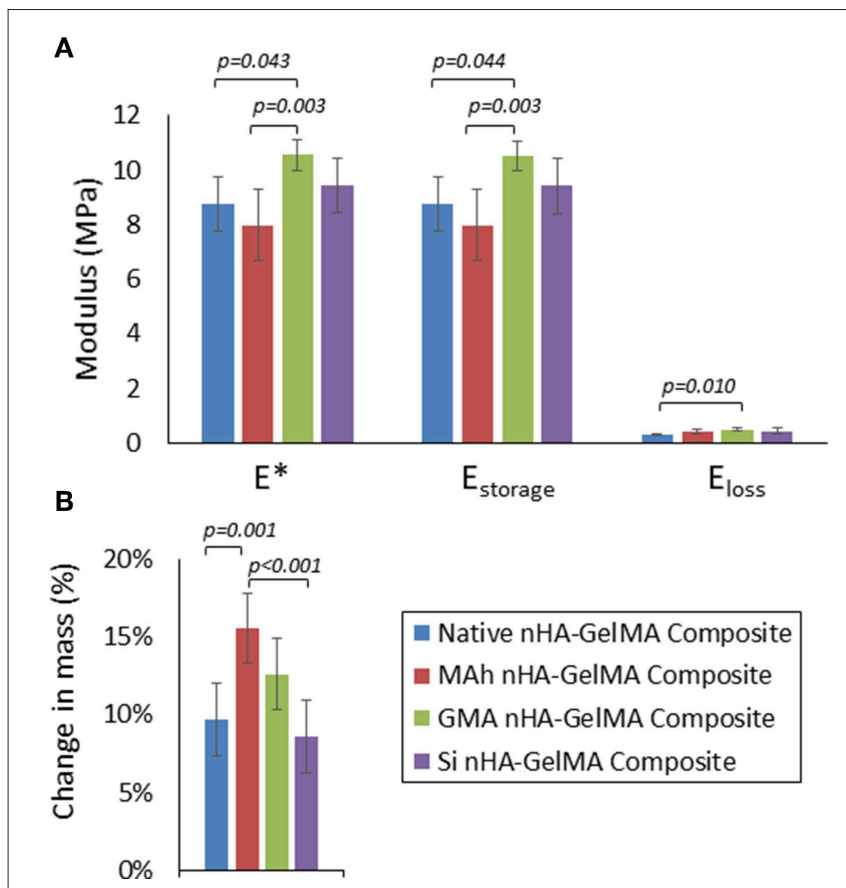

FIGURE 9 | (A) Dynamic stiffness $\left(E^{*}\right)$, storage modulus ( $\left.E_{\text {storage }}\right)$, and loss modulus ( $\left.E_{\text {loss }}\right)$, and $(\mathbf{B})$ change in mass after $3 \mathrm{~d}$ in $37^{\circ} \mathrm{C}$ water for the nHA-GelMA composites. In this study $\mathrm{nHA}$ was methacrylated using methacrylic anhydride (MAh) or glycidyl methacrylate (GMA); silanated $\mathrm{nHA}$ is represented by Si-nHA. Horizontal bars indicate statistically detectable difference ( $p$-values indicated) $(n=6)$.

gravity settling experiments revealed a small dependence on methacrylate type after $24 \mathrm{~h}$ in ethanol; both native and GMA-modified nHA particles dispersed better in ethanol than the MAh- or Si-modified nHA particles. This latter result corroborated well with the detectably greater magnitude of zeta potential reported for native and GMA-nHA in ethanol (compared to the other sample groups). Finally, a proofof-concept investigation revealed that a GMA-nHA-GelMA composite had a greater dynamic stiffness than the native nHA-GelMA composite, and comparable swelling after $3 \mathrm{~d}$ in water.

By analyzing the dependence of nHA surface methacrylation on the type of methacrylate agent, this study has improved

\section{REFERENCES}

Amdjadi, P., Ghasemi, A., Najafi, F., and Nojehdehian, H. (2017). Pivotal role of filler matrix interface in dental composites: review. Biomed. Res. 28, 1054-1065.

Arcís, R. W., López-Macipe, A., Toledano, M., Osorio, E., RodríguezClemente, R., Murtra, J., et al. (2002). Mechanical properties of visible light-cured resins reinforced with hydroxyapatite for dental restoration. Dental Mater. 18, 49-57. doi: 10.1016/S0109-5641(01) 00019-7

Avci, D., and Mathias, L. J. (2005). Synthesis and photopolymerizations of phosphate-containing acrylate/(di)methacrylate monomers from 3(acryloyloxy)-2-hydroxypropyl methacrylate. Polymer Bull. 54, 11-19. doi: 10.1007/s00289-005-0357-6 current understanding of non-stoichiometric nHA surface affinity for methacrylates. This study also presents a gentler approach to methacrylation of the HA surface and introduction of vinyl groups. Lastly, this work highlights the significance of methacrylate reagent on non-stoichiometric nHA dispersion, with GMA-modified nHA particles showing a notable improvement in ethanol dispersion compared to MAhmodified nHA. As a result, the GMA-modified nHA particles are a viable alternative to silanated $\mathrm{nHA}$ in the fabrication of future biomimetic composites, owing to improved dispersion in ethanol and similar covalent nature of the nHA surface modification.

\section{DATA AVAILABILITY STATEMENT}

The datasets generated and analyzed during the current study are available from the corresponding author upon reasonable request.

\section{AUTHOR CONTRIBUTIONS}

PC conceived and conducted the experiments, analyzed and interpreted the results, and was the primary author of the manuscript. TW acquired the research operating funding, advised on the conception and conduction of the experiments and data analysis, and assisted in the editing and revisions of the manuscript as the senior author.

\section{FUNDING}

The authors would like to thank the Natural Sciences and Engineering Research Council of Canada for their support in the form of a postdoctoral fellowship (PC) and a Discovery Grant (No. 3942, TW). This study was also partially supported by a grant from the Canadian Institutes of Health Research (No. 389569, TW).

\section{ACKNOWLEDGMENTS}

We are grateful for the assistance of Jan Venne in the collection of the NMR data reported in this study, and Danielle Erb for her assistance in collecting dynamic stiffness data. The authors also thank Professor Leonardo Simon (University of Waterloo) for helpful discussions.

Cheng, K., Hirose, M., Wang, X., Sogo, Y., Yamazaki, A., and Ito, A. (2013). Correlation between cell attachment areas after $2 \mathrm{~h}$ of culture and osteogenic differentiation activity of rat mesenchymal stem cells on hydroxyapatite substrates with various surface properties. Biochem. Biophys. Res. Commun. 430, 156-160. doi: 10.1016/j.bbrc.2012.11.054

Chesnut, D. B., Wright, D. W., and Krizek, B. A. (1988). NMR chemical shifts and intramolecular van Der Waals interactions: carbonyl and ether systems. J. Mol. Struct. 190, 99-111. doi: 10.1016/0022-2860(88)80275-8

Cisneros-Pineda, O. G., Herrera Kao, W., Loría-Bastarrachea, M. I., VeranesPantoja, Y., Cauich-Rodríguez, J. V., and Cervantes-Uc, J. M. (2014). Towards optimization of the silanization process of hydroxyapatite for its use in bone cement formulations. Mater. Sci. Eng. C 40, 157-163. doi: 10.1016/j.msec.2014.03.064 
Comeau, P., and Willett, T. (2018). Impact of side chain polarity on nonstoichiometric nano-hydroxyapatite surface functionalization with amino acids. Sci. Rep. 8:12700. doi: 10.1038/s41598-018-31058-5

El Shafei, G. M., and Moussa, N. A. (2001). Adsorption of some essential amino acids on hydroxyapatite. J. Colloid Interface Sci. 238, 160-166. doi: $10.1006 /$ jcis.2001.7474

El-Ghannam, A. (2005). Bone reconstruction: from bioceramics to tissue engineering. Expert Rev. Med. Devices 2, 87-101. doi: 10.1586/17434440.2.1.87

Figueiredo, M., Gamelas, J., and Martins, A. (2012). "Characterization of bone and bone-based graft materials using FTIR spectroscopy," in Infrared Spectroscopy-Life and Biomedical Sciences, eds T. Theophile (Rijeka: InTech), 315-38. doi: 10.5772/intechopen.68547

Frame, J. W., Browne, R. M., and Brady, C. L. (1981). Hydroxyapatite as a bone substitute in the jaws. Biomaterials 2, 19-22. doi: 10.1016/0142-9612(81)90082-X

Gamelas, J. A. F., and Martins, A. G. (2015). Surface properties of carbonated and non-carbonated hydroxyapatites obtained after bone calcination at different temperatures. Colloids Surfaces A Physicochem. Eng. Aspects 478, 62-70. doi: 10.1016/j.colsurfa.2015.03.044

Gonzalez-McQuire, R., Chane-Ching, J. Y., Vignaud, E., Lebugle, A., and Mann, S. (2004). Synthesis and characterization of amino acidfunctionalized hydroxyapatite nanorods. J. Mater. Chem. 14, 2277-2281. doi: 10.1039/b400317a

Halvorson, R. H., Erickson, R. L., and Davidson, C. L. (2003). The effect of filler and silane content on conversion of resin-based composite. Dental Mater. 19, 327-333. doi: 10.1016/S0109-5641(02)00062-3

Jahromi, M. T., Yao, G., and Cerruti, M. (2013). The importance of amino acid interactions in the crystallization of hydroxyapatite. J. R. Soc. Interface R. Soc. 10:20120906. doi: 10.1098/rsif.2012.0906

Ji, X., Hampsey, J. E., Hu, Q., He, J., Yang, Z., and Lu, Y. (2003). Mesoporous silica-reinforced polymer nanocomposites. Chem. Mater. 15, 3656-3662. doi: $10.1021 / \mathrm{cm} 0300866$

Lee, H. J., Choi, H. W., Kim, K. J., and Lee, S. C. (2006). Modification of hydroxyapatite nanosurfaces for enhanced colloidal stability and improved interfacial adhesion in nanocomposites. Chem. Mater. 18, 5111-5118. doi: $10.1021 / \mathrm{cm} 061139 \mathrm{x}$

Leite Ferreira, B. J. M., Barroca, N. B., Lopes, P. R., Daniel-da-Silva, A. L., Helena, M., and Correla, R. N. (2014). Properties of novel PMMA-Co-EHA bone cements filled with hydroxyapatite. Polymers Polymer Composites 35, 759-767. doi: $10.1002 / \mathrm{pc}$

Li, S., and Chesnut, D. B. (1986). Intramolecular van Der Waals interactions and ${ }^{13} \mathrm{C}$ chemical shifts: substituent effects in some cyclic and bicyclic systems. Magn. Resonance Chem. 24, 93-100. doi: 10.1002/mrc.1260240202

Liu, F., Wang, R., Cheng, Y., Jiang, X., Zhang, Q., and Zhu, M. (2013). Polymer grafted hydroxyapatite whisker as a filler for dental composite resin with enhanced physical and mechanical properties. Mater. Sci. Eng. C 33, 4994-5000. doi: 10.1016/j.msec.2013.08.029

Liu, H., Jiang, W., and Malshe, A. (2009). Novel Nanostructured Hydroxyapatite Coating for Dental and Orthopedix Implants. JOM 61, 67-69. doi: 10.1007/s11837-009-0137-0

Liu, Q., Ding, J., Chambers, D. E., Debnath, S., Wunder, S. L., and Baran, G. R. (2001). Filler-coupling agent-matrix interactions in silica/polymethylmethacrylate composites. J. Biomed. Mater. Res. 57, 384-393. doi: 10.1002/1097-4636(20011205)57:3<384::AID-JBM1181>3.0.CO;2-F

Liyanage, D. D., Thamali, R. J. K. A., Kumbalatara, A. A. K., Weliwita, J. A., and Witharana, S. (2016). An analysis of nanoparticle settling times in liquids. J. Nanomater. 2016:7061838. doi: 10.1155/2016/7061838

Logtenberg, E. H. P., and Stein, H. N. (1986). Zeta potential and coagulation of $\mathrm{ZnO}$ in alcohols. Colloids Surfaces 17, 305-312. doi: 10.1016/0166-6622(86)80254-2

Lung, C. Y., Sarfraz, Z., Habib, A., Khan, A. S., and Matinlinna, J. P. (2016). Effect of silanization of hydroxyapatite fillers on physical and mechanical properties of a Bis-GMA based resin composite. J. Mech. Behav. Biomed. Mater. 54, 283-294. doi: 10.1016/j.jmbbm.2015.09.033

Mertz, G., Fouquet, T., Becker, C., Ziarelli, F., and Ruch, D. (2014). A methacrylic anhydride difunctional precursor to produce a hydrolysis-sensitive coating by aerosol-assisted atmospheric plasma process. Plasma Processes Polymers 11, 728-733. doi: 10.1002/ppap.201400050

Murugan, R., and Panduranga Rao, K. (2003). Grafting of glycidyl methacrylate upon coralline hydroxyapatite in conjugation with demineralized bone matrix using redox initiating system. Macromol. Res. 11, 14-18. doi: 10.1007/BF03218272

Murugan, R., and Ramakrishna, S. (2004). Coupling of therapeutic molecules onto surface modified coralline hydroxyapatite. Biomaterials 25, 3073-3080. doi: 10.1016/j.biomaterials.2003.09.089

Pieters, I. Y., Van den Vreken, N. M., Declercq, H. A., Cornelissen, M. J., and Verbeeck, R. M. (2010). Carbonated apatites obtained by the hydrolysis of monetite: influence of carbonate content on adhesion and proliferation of MC3T3-E1 osteoblastic cells. Acta Biomater. 6, 1561-1568. doi: 10.1016/j.actbio.2009.11.002

Poralan, G. M., Gambe, J. E., Alcantara, E. M., and Vequizo, R. M. (2015). X-ray diffraction and infrared spectroscopy analyses on the crystallinity of engineered biological hydroxyapatite for medical application. IOP Conf. Ser. Mater. Sci. Eng. 79:012028. doi: 10.1088/1757-899X/79/1/012028

Redey, S. A., Razzouk, S., Rey, C., Bernache-Assollant, D., Leroy, G., Nardin, M., et al. (1999). Osteoclast adhesion and activity on synthetic hydroxyapatite, carbonated hydroxyapatite, and natural calcium carbonate: relationship to surface energies. J. Biomed. Mater. Res. 45, 140-147. doi: 10.1002/(SICI)10974636(199905)45:2<140::AID-JBM9>3.0.CO;2-I

Reis, A. V., Reis, A. V., Rubira, A. F., and Muniz, E. C. (2009). Reaction of glycidyl methacrylate at the hydroxyl and carboxylic groups of poly(vinyl alcohol) and poly(acrylic acid): is this reaction mechanism still unclear? J. Org. Chem. 74, 3750-3757. doi: 10.1021/jo900033c

Roether, J. A., and Deb, S. (2004). The effect of surface treatment of hydroxyapatite on the properties of a bioactive bone cement. J. Mater. Sci. Mater. Med. 15, 413-418. doi: 10.1023/B:JMSM.0000021112.51065.40

Salopek, B., Krasic, D., and Filipovic, S. (1992). Measurement and application of zeta-potential. Rudarsko-Geolosko-Naftni Zbornik 4, 147-151.

Sarig, S. (2004). Aspartic acid nucleates the apatite crystallites of bone: a hypothesis. Bone 35, 108-113. doi: 10.1016/j.bone.2004.02.020

Temenoff, J. S., Shin, H., Conway, D. E., Engel, P. S., and Mikos, A. G. (2003). In vitro cytotoxicity of redox radical initiators for cross-linking of oligo(poly(ethylene glycol) fumarate) macromers. Biomacromolecules 4, 1605-1613. doi: 10.1021/bm030056w

Tkalčec, E., Popović, J., Orlić, S., Milardović, S., and Ivanković, H. (2014). Hydrothermal synthesis and thermal evolution of carbonatefluorhydroxyapatite scaffold from cuttlefish bones. Mater. Sci. Eng. C 42, 578-586. doi: 10.1016/j.msec.2014.05.079

Valle, L. J., Bertran, O., Chaves, G., Revilla-López, G., Rivas, M., Casas, M. T., et al. (2014). DNA adsorbed on hydroxyapatite surfaces. J. Mater. Chem. B 2, 6953-6966. doi: 10.1039/c4tb01184h

Vaz, C. M., Reis, R. L., and Cunha, A. M. (2002). Use of coupling agents to enhance the interfacial interactions in starch-EVOH/hydroxylapatite composites. Biomaterials 23, 629-635. doi: 10.1016/S0142-9612(01) 00150-8

White, B., Banerjee, S., O’Brien, S., Turro, N. J., and Herman, I. P. (2007). Zeta-potential measurements of surfactant-wrapped individual single-walled carbon nanotubes. J. Phys. Chem. C 111, 13684-13690. doi: 10.1021/jp0 $70853 e$

Witharana, S., Hodges, C., Xu, D., Lai, X., and Ding, Y. (2012). Aggregation and settling in aqueous polydisperse alumina nanoparticle suspensions. $J$. Nanoparticle Res. 14, 1-19. doi: 10.1007/s11051-012-0851-3

Xie, X. L., Tang, C. Y., Zhou, X. P., Li, R. K. Y., Yu, Z. Z., Zhang, Q. X., et al. (2004). Enhanced interfacial adhesion between PPO and glass beads in composites by surface modification of glass beads via in situ polymerization and copolymerization. Chem. Mater. 16, 133-138. doi: 10.1021/cm034512z

Zou, Z., Liu, X., Chen, L., Lin, K., and Chang, J. (2012). Dental enamel-like hydroxyapatite transformed directly from monetite. J. Mater. Chem. 22:22637. doi: $10.1039 / \mathrm{c} 2 \mathrm{jm} 35430 \mathrm{f}$

Conflict of Interest: The authors declare that the research was conducted in the absence of any commercial or financial relationships that could be construed as a potential conflict of interest.

Copyright (c) 2019 Comeau and Willett. This is an open-access article distributed under the terms of the Creative Commons Attribution License (CC BY). The use, distribution or reproduction in other forums is permitted, provided the original author(s) and the copyright owner(s) are credited and that the original publication in this journal is cited, in accordance with accepted academic practice. No use, distribution or reproduction is permitted which does not comply with these terms. 